\title{
Role of ketohexokinase in the endothelium-dependent relaxation of thoracic aorta from diabetic model mouse
}

\section{Mayumi Mori-Kawabe ${ }^{1}$, Tomohito Doke ${ }^{2}$, Takahiro Hayasaki ${ }^{2}$, Takuji Ishimoto ${ }^{2}$, Akiyoshi Hirayama $^{3}$, Tomoyoshi Soga ${ }^{3}$}

\author{
${ }^{I}$ Department of Pharmacology, Nagoya City University Graduate School of Medical Sciences, Japan, ${ }^{2}$ Department of \\ Nephrology, Nagoya University Graduate School of Medicine, Japan, ${ }^{3}$ Institute for Advanced Biosciences, Keio \\ University, Japan
}

Background: The metabolism of fructose and its consequent activation of nucleotide degradation pathway have been reported to correlate with metabolic syndrome and diabetes. However, it is unclear how fructose metabolism affects vascular response. Fructose is primarily metabolized by ketohexokinase (KHK) which have two isoforms, KHK-C and KHK-A. In this work, we tested aortic responsibility in KHK-knockout (KO) diabetic model mice, investigating mechanism for the relationship between fructose metabolism and vascular function.

Methods: Diabetes was induced by low-dose streptozotocin in male wild-type (WT), KHK-A KO, and KHK-A/C KO mice lacking both isoforms. After 24 weeks feeding with high fat diet, biochemical analysis of blood and metabolomics of urine and kidney tissues were carried out. Blood pressure was measured by tail cuff method. Urinary nitrate/nitrite concentration was measured using a Colorimetric Assay Kit. Mechanical activity of thoracic aortas from these mice was measured by isometric recording. Acetylcholine (ACh) - or sodium nitroprusside (SNP) -induced relaxation, and the effects of NO synthase inhibitor, N-nitro-L-arginine (LNA), on the contracting effect of phenylephrine (PE) were examined using endothelium-intact aortas from the mice.

Results: The levels of body weight, blood glucose and blood pressure were similar among the diabetic mice. However, urinary nitrate/nitrite concentration was significantly lower in diabetic WT and diabetic KHK-A KO compared with diabetic KHK-A/C KO. Although ACh or SNP caused concentration-dependent relaxation on the endothelium-intact aortas, the relaxing effect was not significantly different among the all diabetic mice. However, the pretreatment with 100 $\mu \mathrm{M}$ LNA significantly decreased the contraction of $10 \mu \mathrm{M}$ PE on endothelium-intact aortas from the diabetic WT and KHK-A KO mice compared with the diabetic KHK-A/C KO. These decreases were inversely correlated with serum xanthine oxidase activity and urinary uric acid.

Conclusions: The vascular function expressed as the effect of LNA was impaired in the diabetic WT and KHK-A KO mice, whereas it was preserved in the diabetic KHK-A/C KO mice, inversely correlating with the levels of serum xanthine oxidase activity and urinary uric acid. The results suggest that the endothelial NO-related dysfunction in high fat fed diabetic mice might be due to KHK-C dependent fructose metabolism. 